Nuclear Physics B222 (1983) 11-19

(C) North-Holland Publishing Company

\title{
FERMION MASSES AND INDUCTION OF THE WEAK SCALE BY SUPERGRAVITY
}

\author{
J.-M. FRĖRE, D.R.T. JONES and S. RABY ${ }^{1}$ \\ Randall Laboratory of Physics, The University of Michigan, Ann Arbor, MI 48109, USA
}

Received 11 January 1983

\begin{abstract}
We discuss some problems which arise in a low-energy theory of strong and electroweak interactions when the weak scale is induced via supergravity. The main problem concerns the origin of quark and lepton masses. If the fermions obtain their masses in the conventional way via Yukawa couplings, then the low-energy supergap (mass difference between supersymmetric partners) is comparable to the mass of the lightest massive fermion. One solution would require that at least the light generations receive their mass indirectly via radiative corrections. Alternatively it might be that the $S U(2) \times U(1)$ breaking is induced radiatively.
\end{abstract}

\section{Introduction}

We want to indicate some problems which arise in low-energy strong and electroweak theories when the weak scale is induced via supergravity. We do not know at present how general these problems may be. Thus it is particularly important that we state our basic assumptions.

We shall consider an $S U(3) \otimes S U(2) \otimes U(1)$ gauge theory of strong and electroweak interactions with local supersymmetry (SUSY). We assume the theory has two distinct sectors coupled only via supergravity. The first is the super Higgs sector of the theory which spontaneously breaks SUSY and gives the gravitino a mass $m_{3 / 2}[1]$. The second includes the gauge fields, quarks, leptons and Higgs. SUSY breaking effects are induced in the second sector by the first, and are of order $m_{3 / 2}$ $[2,3]$.

We assume that these effects are the sole driving terms for the weak breaking scale $v$ of order $\boldsymbol{M}_{\mathrm{w}}$ [4]. We thus require that the only dimensionful parameters in the low-energy theory be induced by the super Higgs sector.

We shall use a superspace potential of the form (we use the notation of Nilles et al., ref. [4]):

$$
W=h\left(Z_{i}\right)+g\left(Y_{a}\right),
$$

where $Z_{i}$ are the fields in the super Higgs sector and $Y_{a}$ include quarks, leptons and Higgs.

\footnotetext{
${ }^{1}$ On leave of absence from Los Alamos National Laboratory, Los Alamos, NM 87545, USA.
} 
The scalar potential is given by [1]

$$
\begin{aligned}
V= & \exp \left[\left(\left|Z_{i}\right|^{2}+\left|Y_{a}\right|^{2}\right) / M^{2}\right]\left[\left|h_{i}+Z_{i}^{*} W / M^{2}\right|^{2}\right. \\
& \left.+\left|g_{a}+Y_{a}^{*} W / M^{2}\right|^{2}-3|W|^{2} / M^{2}\right]+\frac{1}{2} D_{a} D_{\alpha},
\end{aligned}
$$

where sums over $i, a$ and $\alpha$ are understood, and

$$
\begin{gathered}
M=M_{P} / \sqrt{8 \pi}=2.4 \times 10^{18} \mathrm{GeV}, \\
h_{i}=\frac{\partial h}{\partial Z_{i}}, \quad g_{a}=\frac{\partial g}{\partial Y_{a}}, \quad D_{\alpha}=e_{\alpha} \sum_{a} Y_{a}^{*} T^{\alpha} Y_{a},
\end{gathered}
$$

where $T^{\alpha}$ is a gauge group generator and $e_{\alpha}$ the associated coupling constant. Following ref. [4] we assume $V$ is minimized when $Z_{i}, h_{i}$ and $h$ have vacuum expectation values

$$
\left\langle Z_{i}\right\rangle=b_{i} M, \quad\left\langle h_{i}\right\rangle=a_{i}^{*} m M, \quad\langle h\rangle=m M^{2},
$$

where $m \ll M$. The low-energy potential $V_{\text {L.E. }}$ is obtained by replacing $Z_{i}, h_{i}$ and $h$ by their VEVs and keeping only those terms which do not vanish as $M \rightarrow \infty$. The result is

$$
V_{\mathrm{L.E.}}=\left|\tilde{g}_{a}\right|^{2}+m_{3 / 2}(A \tilde{g}+\text { h.c. })+m_{3 / 2}^{2}\left|Y_{a}\right|^{2}+\frac{1}{2} D_{\alpha} D_{\alpha},
$$

where $m_{3 / 2}$ is the gravitino mass given by

$$
m_{3 / 2}=\exp \left(\frac{1}{2}\left|b_{i}\right|^{2}\right) m,
$$

$\tilde{g}$ is the rescaled superpotential

$$
\tilde{g}=\exp \left(\frac{1}{2}\left|b_{i}\right|^{2}\right) g,
$$

and the constant $A$ is given by

$$
A=b_{i}^{*}\left(a_{i}+b_{i}\right) .
$$

In obtaining this result we have assumed the following.

(i) The potential $V$ vanishes at the minimum. Hence there is no cosmological constant. This requires

$$
\sum_{i}\left|a_{i}+b_{i}\right|^{2}=3 .
$$

After minimizing $V_{\text {L.E. }}$ we shall in general obtain additional contributions to the cosmological constant of order $m_{3 / 2}^{4}$. This will require additional fine tuning of relation (1.10) by small corrections of order $m_{3 / 2}^{2} / M^{2}$. We have

(ii) used the assumption that $g\left(Y_{a}\right)$ contains no dimensionful parameters which allows use of the identity

$$
Y_{a} \tilde{g}_{a}=3 \tilde{g}
$$


One immediate (and attractive) consequence of the philosophy espoused here is apparent from (1.6); all scalars obtain identical masses of $\mathrm{O}\left(m_{3 / 2}\right)$. Thus the GIM cancellation required to avoid awkward flavor-changing neutral currents is automatic.

\section{Some simple models}

Let us now consider a simple example in which the weak scale is induced by supergravity. Let

$$
\tilde{g}=\lambda Y \bar{H}^{i} H_{i},
$$

where $Y$ is an $\mathrm{SU}(2) \otimes \mathrm{U}(1)$ singlet; $\bar{H}^{i}$ and $H_{i}$ are Higgs doublets and $i=1,2$ is an SU(2) index. $\lambda$ is an arbitrary Yukawa coupling which we shall without loss of generality take to be real and positive. In an obvious notation we identify the Higgs content by

$$
\bar{H}^{i}=\left(\begin{array}{c}
\bar{H}^{-} \\
\bar{H}^{\circ}
\end{array}\right), \quad H_{i}=\left(\begin{array}{c}
H^{+} \\
H^{\circ}
\end{array}\right) .
$$

The low-energy potential $V_{\text {L.E. }}$ is given by

$$
\begin{aligned}
V_{\text {L.E. }}= & \lambda^{2}\left(\left|\bar{H}^{\circ}\right|^{2}\left|H^{\circ}\right|^{2}+|Y|^{2}\left|\bar{H}^{\circ}\right|^{2}+|Y|^{2}\left|H^{+}\right|^{2}+|Y|^{2}\left|H^{\circ}\right|^{2}\right) \\
& +m_{3 / 2} \lambda|A|\left(\mathrm{e}^{i \alpha} Y \bar{H}^{\circ} H^{\circ}+\text { h.c. }\right)+m_{3 / 2}^{2}\left(|Y|^{2}+\left|\bar{H}^{\circ}\right|^{2}+\left|H^{\circ}\right|^{2}+\left|H^{+}\right|^{2}\right) \\
& +\frac{1}{8} g_{2}^{2}\left[4\left|H^{+}\right|^{2}\left|H^{\circ}\right|^{2}+\left(\left|H^{+}\right|^{2}-\left|H^{\circ}\right|^{2}+\left|\bar{H}^{\circ}\right|^{2}\right)^{2}\right]+\frac{1}{8} g_{1}^{2}\left(\left|H^{+}\right|^{2}+\left|H^{\circ}\right|^{2}-\left|\bar{H}^{\circ}\right|^{2}\right)^{2},
\end{aligned}
$$

where we have already used the SU(2) invariance of $V_{\text {L.E. }}$ to set $\bar{H}^{-} \equiv 0$. We then immediately see that at the minimum of $V_{\text {L.E. }}, H^{+}=0$ and electric charge is conserved.

Let's now define $Y=y \mathrm{e}^{i \theta_{y}}, \bar{H}^{\circ}=\bar{h}^{\circ} \mathrm{e}^{i \bar{\theta}_{0}}$ and, $H^{\circ}=h^{\circ} \mathrm{e}^{i \theta_{0}}$ where $y, \bar{h}^{\circ}, h^{\circ}$ are real and positive. The global minium of $V_{\text {L.E. }}$ depends on $A$, and is given by:

$$
\begin{aligned}
& \text { for }|A|<3, \quad y=\bar{h}^{\circ}=h^{\circ}=0, \quad V_{\text {L.E. }}(0)=0, \\
& \text { or for }|A|>3, \quad y=\bar{h}^{\circ}=h^{\circ} \equiv u \frac{m_{3 / 2}}{\lambda}, \\
& \quad \theta_{y}+\bar{\theta}_{0}+\theta_{0}+\alpha=\pi, \quad(\bmod 2 \pi),
\end{aligned}
$$

where

$$
\begin{gathered}
u=\frac{1}{4}\left(|A|+\sqrt{|A|^{2}-8}\right)>1, \\
V_{\text {L.E. }}(\mathrm{u}) \equiv-\frac{m_{3 / 2}^{4}}{\lambda^{2}}\left(u^{2}-1\right) u^{2}
\end{gathered}
$$




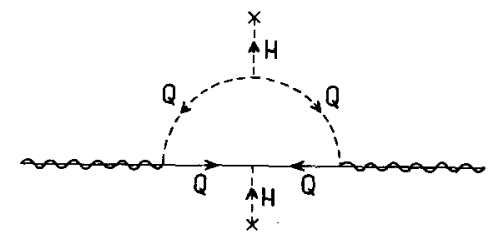

Fig. 1. A one-loop contribution to the gluino mass.

We shall henceforth assume that there exists a super Higgs sector $h\left(Z_{i}\right)$ for which $|A|>3$. Note that it was shown in ref. [4] that the condition $|A|>3$ is in general necessary to induce the weak scale. In our simple model it is also a sufficient condition.

The $\theta^{2}$ component of the superspace potential $\tilde{g}=\lambda Y \bar{H}^{i} H_{i}$ is invariant under three global symmetries:

(i) $\quad Y(x, \theta) \rightarrow \mathrm{e}^{-i_{3}^{2} \alpha} Y\left(x, \theta \mathrm{e}^{i \alpha}\right)$ and similar transformations for $\bar{H}, H$

(ii) $Y \rightarrow \mathrm{e}^{2 i \theta} Y, \quad \bar{H}^{i} \rightarrow \mathrm{e}^{-i \theta} \bar{H}^{i}, \quad H_{i} \rightarrow \mathrm{e}^{-i \theta} H_{i}$,

(iii) $\quad Y \rightarrow Y, \quad \bar{H}^{i} \rightarrow \mathrm{e}^{i \theta} \bar{H}^{i}, \quad H_{i} \rightarrow \mathrm{e}^{-i \theta} H_{i}$.

The symmetry (i) is an $R$ symmetry which may or may not be a symmetry of the full superspace potential $W$. It is also either spontaneously or explicitly broken by the super Higgs sector. As a result it is clearly not a symmetry of $V_{\text {L.E. }}$.

The fact that the cubic terms proportional to $A$ violate $R$ symmetry and supersymmetry means that gauginos develop masses in perturbation theory.

At the one-loop level we estimate (see fig. 1) a gluino mass

$$
\sim \frac{A \alpha_{\mathrm{s}}}{\pi} \frac{m_{\mathrm{t}}^{2}}{m_{3 / 2}} \sim 0.15 A\left(\frac{m_{\mathrm{t}}}{20}\right)^{2} \mathrm{GeV}
$$

where $m_{\mathrm{t}}$ is the mass of the heaviest quark (assuming $m_{\mathrm{t}}<m_{3 / 2}$ ). At the two-loop level ${ }^{\star}$, however, we encounter fig. 2 , which gives a logarithmically divergent contribution

$$
\sim A \frac{\alpha_{\mathrm{s}} \alpha}{\pi^{2} \sin ^{2} \theta_{\mathrm{w}}}\left(\frac{m_{\mathrm{t}}}{m_{\mathrm{w}}}\right)^{2} m_{3 / 2} \ln \Lambda
$$

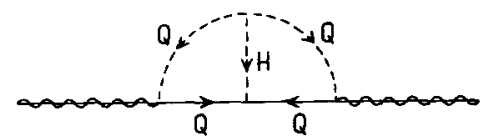

Fig. 2. A (divergent) two-loop contribution to the gluino mass.

* This effect has been independently observed in a slightly different context by Aulakh and Mohapatra [5]. 
where $\Lambda$ is the cut-off. If we put $\Lambda \sim M_{\mathrm{P}}$ then this contribution is not larger than the one-loop one; however the presence of the divergence indicates a sensitivity to unknown states at higher masses. If we wish our low-energy description to correspond to a renormalizable field theory we must include bare mass terms for the gauginos and regard their masses as free parameters of the theory.

We can see that (ii) is a symmetry of $V_{\text {L.E. }}$ which is spontaneously broken at the weak scale and results in an unwanted Peccei-Quinn axion. Finally (iii) is the local $\mathrm{U}(1)$ weak hypercharge.

In order for our simple model to be phenomenologically acceptable we must explicitly break the global symmetry (ii). We thus define an improved $\tilde{g}^{\prime}$ given by

$$
\tilde{g}^{\prime}=\frac{1}{3} \sigma Y^{3}+\lambda Y \bar{H}^{i} H_{i} .
$$

(ii) is now broken and the axion obtains mass $m_{\mathrm{a}}$ of order

$$
m_{\mathrm{a}} \sim\left(\frac{\sigma}{\lambda}\right)^{1 / 2} m_{3 / 2}
$$

We can now repeat our previous analysis on $\tilde{g}^{\prime}$ and the new $V_{\text {L.E. }}^{\prime}$ We find for $0<\sigma \ll \lambda$ that the global minimum is at $y \neq 0, h^{\circ}=\bar{h}^{\circ}=0$. If however $\sigma / \lambda \geqslant 1$ then there exists a solution for $y \neq 0$ and

$$
h^{\circ}=\bar{h}^{\circ} \simeq \frac{m_{3 / 2}}{\lambda^{\prime}} A,
$$

and the energy in the potential at the minimum is of order

$$
\left.V_{\text {L.E. }}^{\prime}\right|_{\min } \simeq-m_{3 / 2}^{4}\left(\lambda^{\prime}\right)^{-2} A^{4},
$$

where

$$
\lambda^{\prime} \equiv \lambda\left(\frac{\lambda}{\sigma}\right)^{1 / 2}
$$

\section{Fermion masses}

We must now include some Higgs couplings to quarks and leptons in order to generate any fermion masses. As a first try let us assume that all quarks and leptons receive their mass at the tree level via Higgs Yukawa couplings. We then have

$$
\tilde{g}=\frac{1}{3} \sigma Y^{3}+\lambda Y \bar{H}^{i} H_{i}+\lambda_{a b}^{\mathrm{u}} H_{i} Q_{j}^{a} \bar{U}^{b} \varepsilon^{i j}+\lambda_{a b}^{\mathrm{d}} \bar{H}^{i} Q_{i}^{a} \bar{D}^{b}+\lambda_{a b}^{e} \bar{H}^{i} L_{i}^{a} \bar{E}^{b},
$$

where $a, b=1,2,3$ are generation indices; $\lambda_{u}, \lambda_{d}$ and $\lambda_{\ell}$ are Yukawa couplings and in an obvious notation $Q_{i}^{1 / 3}\left(L_{i}^{-1}\right)$ and $\bar{U}^{-4 / 3}, \bar{D}^{2 / 3}\left(\bar{E}^{2}\right)$ are respectively the quark (lepton) SU(2) doublets and singlets. Their weak hypercharge is explicitly indicated. 
We can now immediately write down the low-energy potential (see 1.6). The relevant $D_{\alpha}$ are given by

$$
\begin{aligned}
& D_{3}^{\mathrm{A}}=\frac{1}{2} g_{3}\left(Q^{*} \lambda^{A} \mathbb{1}_{2} Q-\bar{U}^{*}\left(\lambda^{A}\right)^{\mathrm{T}} \bar{U}-\bar{D}^{*}\left(\lambda^{A}\right)^{\mathrm{T}} \bar{D}\right), \quad A=1, \ldots, 8, \\
& D_{2}^{I}=\frac{1}{2} g_{2}\left(Q^{*} \rrbracket_{3} \tau^{I} Q+L^{*} \tau^{I} L+H^{*} \tau^{I} H-\bar{U}^{*}\left(\tau^{I}\right)^{\mathrm{T}} \bar{H}\right), \quad I=1, \ldots, 3, \\
& D_{1}=\frac{1}{2} g_{1}\left(\frac{1}{3} Q^{*} \rrbracket_{3} \rrbracket_{2} Q+\frac{2}{3} \bar{D}^{*} \rrbracket_{3} \bar{D}-\frac{4}{3} \bar{U}^{*} \mathbb{1}_{3} \bar{U}-L^{*} \rrbracket_{2} L+2 \bar{E}^{*} \bar{E}+H^{*} \mathbb{1}_{2} H-\bar{H}^{*} \mathbb{1}_{2} \bar{H}\right) .
\end{aligned}
$$

$V_{\text {L.E. }}$ is extremely complicated. However it is not too difficult to convince oneself of the following two facts.

(i) if $\lambda_{\mathrm{u}}, \lambda_{\mathrm{d}}$ and $\lambda_{\ell} \ll \lambda^{\prime} \simeq 1$, then there exists a local minimum of $V_{\text {L.E. }}$ with VEVs given by (2.8) and all other VEVs vanishing. This is the desired solution.

(ii) However, the global minimum of $V_{\text {L.E. }}$ in the same range of parameters $\lambda_{u}$, $\lambda_{\mathrm{d}}$ and $\lambda_{\ell} \ll \lambda^{\prime} \simeq 1$ spontaneously breaks at least electric charge invariance with the scalar partners of the lightest quark or lepton pair obtainng a non-vanishing VEV.

Consider for example a much simplified superspace potential $\tilde{g}$ which will make the second statement obvious. Let

$$
\tilde{g}=\frac{1}{3} \sigma Y^{3}+\lambda Y \bar{H} H+\lambda_{\mathrm{e}} \bar{H} L \bar{e},
$$

where $L_{i}=\left(\begin{array}{l}\nu \\ e\end{array}\right)$. The global minimum of the resultant $V_{\text {L.E. }}$ is located at the point

$$
\left|\bar{H}^{\circ} \|=\right| e|=| \bar{e} \mid=\frac{m_{3 / 2}}{\lambda_{\mathrm{e}}} u,
$$

where $u$ is defined in eq. (2.4) and all other VEVs vanish. The ground state energy is given by

$$
V_{\text {L.E. }}(u)=-\frac{m_{3 / 2}^{4}}{\lambda_{\mathrm{e}}^{2}}\left(u^{2}-1\right) u^{2}
$$

This is clearly lower energy than the desired ground state (2.8).

Note that the $D_{\alpha}$ terms (eq. 3.2) vanish identically along a line defined by (3.4) with an arbitrary scale factor $u$. Thus they do not stabilize scalar quark and lepton VEVs at zero.

The problem is a direct consequence of the small Yukawa couplings $\lambda_{\mathrm{u}}, \lambda_{\mathrm{d}}, \lambda_{\ell} \ll$ $\lambda^{\prime} \simeq 1$ for the light fermion generations. Can we avoid the problem by choosing a different range of parameters i.e. $\lambda^{\prime}<\lambda_{\mathrm{u}}, \lambda_{\mathrm{d}}, \lambda_{\ell}$ ? In that case the extremum (2.8) would be the global minimum of $V_{\text {L.E. }}$. It is easy to see that this range of parameters is not phenomenologically viable. Quark and lepton masses are given by the expression

$$
m_{\mathrm{u}}\left(m_{\mathrm{d}}, m_{\ell}\right)=\lambda_{\mathrm{u}}\left(\lambda_{\mathrm{d}}, \lambda_{\ell}\right) v,
$$

where $v=\left\langle H^{\circ}\right\rangle=\left\langle\bar{H}^{\circ}\right\rangle$. The gravitino mass is given by

$$
m_{3 / 2} \simeq \lambda^{\prime} v .
$$


The gravitino is thus one of the lightest fermions in the theory. Note this is not necessarily a problem, by itself. It is a problem because the gravitino mass is an upper bound for the supergap in the low-energy theory. As such $m_{3 / 2}<m_{\mathrm{u}}, m_{\mathrm{d}}$ or $m_{\ell}$ is experimentally ruled out for all known fermions. We are thus stuck with $\lambda_{\mathrm{u}}, \lambda_{\mathrm{d}}, \lambda_{\ell} \ll \lambda^{\prime} \simeq 1$.

Do radiative corrections destablize the undesirable vacuum (3.4)? Let us consider the one-loop corrections to the model defined by

$$
\tilde{g}=\lambda_{\mathrm{e}} \bar{H} L \bar{e} .
$$

These are given by the usual expression

$$
V_{1}=\frac{1}{64 \pi^{2}} \operatorname{Str} m^{4} \ln \frac{m^{2}}{\mu^{2}},
$$

where $m^{2}$ is the mass matrix and

$$
\text { Str }=\sum_{\text {scalars }}+3 \sum_{\text {vectors }}-2 \sum_{\text {fermions }} .
$$

We would like to evaluate this expression for the vacuum expectation values (3.4) and see whether it is small compared to the tree expression (3.5).

Now $\mathrm{m}^{2}$ for the scalars, for instance, is given (schematically) by

$$
m^{2} \sim m_{3 / 2}^{2}+A m_{3 / 2} \lambda_{e} \phi+\lambda_{\mathrm{e}}^{2} \phi^{2}+g^{2} \phi^{2},
$$

where $\phi$ stands for $(\bar{H}, L, \bar{e})$. (Group indices suppressed) For $\phi \sim m_{3 / 2} / \lambda_{\mathrm{e}}$ the last term is potentially large. However we know that if we set $m_{3 / 2}=\lambda_{e}=0$ then (3.9) will give $V_{1}=0$ for any $\phi$ as long as $D=0$ (which we ensure by $\bar{H}^{\circ}=|e|=|\bar{e}|$. Thus one can explicitly verify that at field values of $\sim m_{3 / 2} / \lambda_{\mathrm{e}}$ the gauge coupling contributes to $V$ a term of at most $g^{2} m_{3 / 2}^{4} / \lambda_{\mathrm{e}}^{2}$. A similar argument holds with respect to possible Yukawa couplings of $H, \bar{H}$ to heavy quarks.

We have not examined in depth the situation in higher orders but believe the above conclusion will not be altered. Thus the minimal model considered above is clearly unsatisfactory. Let us now consider some possible ways out.

We may try to discourage the electron from developing a VEV by adding further couplings. Consider for instance

$$
\tilde{g}=\frac{1}{3} \sigma Y^{3}+\lambda Y \bar{H} H+\lambda_{\mathrm{e}} \bar{H} L \bar{e}+\lambda_{\mathrm{K}} \bar{K} L \bar{e},
$$

where $\bar{K}$ is a weak doublet with the same quantum numbers as $\bar{H}$. Now if we arrange

$$
\lambda_{\mathrm{e}} \ll \lambda^{\prime}<\lambda_{\mathrm{K}},
$$

then the vacuum (2.6) is favored again. This, however, causes phenomenological problems in that, for instance $\mathrm{K}$-exchange in purely leptonic processes is of order

$$
\mathrm{O}\left(\frac{\lambda_{\mathrm{K}}^{2}}{m_{3 / 2}^{2}}\right) \sim \mathrm{O}\left(\frac{\lambda_{\mathrm{K}}^{2}}{\lambda^{\prime 2}} \frac{g^{2}}{m_{\mathrm{w}}^{2}}\right),
$$

which by assumption is stronger than standard weak processes. 
We can get around this problem by introducing a new field $K$ and adding a further term

$$
\lambda_{\mathrm{K}}^{\prime} \bar{K} K H,
$$

which gives a large mass to the $\mathrm{K}$ fields if $\lambda_{\mathrm{K}}^{\prime}>\lambda_{\mathrm{K}}$. However since, as already mentioned, we require $\lambda^{\prime} \sim 1$ to produce a phenomenologically acceptable supergap, tinkering along these lines probably leads to loss of perturbative calculability.

Such a large coupling $\lambda_{K}^{\prime}$ could be avoided by coupling $K$ and $\bar{K}$ to a new field transforming like a singlet under the weak interactions, and capable of developing a vacuum expectation value greater than $h^{\circ}$. In the present framework, this would, however, be quite artificial, and is best qualified as a need to reintroduce the full grand unification framework.

\section{Conclusions}

We have examined a situation where a low-energy supersymmetric $\operatorname{SU}(2) \times U(1)$ lagrangian is softly broken by scalar masses and cubic couplings, originating through a coupling to a hidden sector, through supergravity.

We have shown, that under the assumption that the coefficient of the cubic term, $|A|$, be $\geqslant 3$, spontaneous symmetry breakdown of the gauge symmetry would occur. The vacuum expectation value is however associated with the superpartner of the lightest massive fermion. In the standard model, this would correspond to the electron and result in spontaneous breaking of electric charge conservation! Electric charge may be preserved at the minimal cost of adding a right-handed neutrino. The model would however remain unacceptable, since the "supergap" (mass difference between ordinary particles and their supersymmetric partners) would be of the order of the neutrino mass! Radiative corrections apparently do not improve the situation.

Compatibility of the model with present experimental knowledge could be restored at the cost of introducing new fields, with the purpose of increasing the usual fermions' supersymmetric quartic self-couplings. There is however no other compelling rationale for this extension inside the low-energy theory.

Other possibilties exist, such as requiring that the light fermions obtain masses from radiative corrections only, thus eliminating the troublesome small couplings $\left(\lambda_{e}, \lambda_{u}, \ldots\right)$. Within the general framework of this paper the most attractive possibility seems however to be to take $|A|<3^{\star}$ and hope to generate the $\mathrm{SU}(2) \times \mathrm{U}(1)$ breaking via radiative corrections. The latter scenario has recently been explored [6].

\section{Note added in proof}

In the above we have only been concerned with the issue of an absolute minimum. We are aware of the fact that in a cosmological context, a metastable state may

\footnotetext{
* The special case $|A|=3$ leads to degenerate vacuua; this possibility has been explored in ref. [7].
} 
have a lifetime sufficiently long so as to be consistent with present day observation. This is particularly relevant in view of the large VEVs which characterize the absolute minimum and which make it unlikely to be the first to be reached from the unbroken state. This dynamical question deserves further study.

\section{References}

[1] E. Cremmer, B. Julia, J. Scherk, S. Ferrara, L. Girardello and P. van Nieuwenhuizen, Nucl. Phys. B147 (1979) 105

[2] E. Cremmer, S. Ferrara, L. Girardello and A. van Proeyen, CERN preprint TH-3312 (1982); Nucl. Phys. B212 (1983) 413

R. Barbieri, S. Ferrara and C.A. Savoy, Phys. Lett. 119B (1982) 243

[3] A.H. Chamseddine, R. Arnowitt and Pran Nath, Phys. Rev. Lett. 49, (1982) 970

[4] H.P. Nilles, M. Srednicki and D. Wyler, CERN preprint TH-3432

[5] C.S. Aulakh and R.N. Mohapatra, City College, New York preprint CCNY/HEP/82/15

[6] L. Ibañez, Nucl. Phys. B218 (1983) 514

L. Alvarez-Gaumé, J. Polchinski and M. Wise, Harvard preprint (82)

J. Ellis, D. Nanopoulos and K. Tamvakis, CERN preprint TH-3418

[7] E. Cremmer, P. Fayet and L. Girardello, Ecole Normale Superieure preprint LPTENS 82/30, 1982 